\title{
Cooper pair dispersion relation for weak to strong coupling
}

\author{
Sadhan K. Adhikari, ${ }^{1}$ M. Casas, ${ }^{2}$ A. Puente,,${ }^{2}$ A. Rigo, ${ }^{2}$ M. Fortes, ${ }^{3}$ M. A. Solís, ${ }^{3}$ M. de Llano, ${ }^{4}$ Ariel A. Valladares, ${ }^{4}$ \\ and O. Rojo ${ }^{5}$ \\ ${ }^{1}$ Instituto de Física Teórica, Universidade Estadual Paulista, 01405-900 São Paulo, SP, Brazil \\ ${ }^{2}$ Departament de Física, Universitat de les Illes Balears, 07071 Palma de Mallorca, Spain \\ ${ }^{3}$ Instituto de Física, Universidad Nacional Autónoma de México, 01000 México, DF, Mexico \\ ${ }^{4}$ Instituto de Investigaciones en Materiales, Universidad Nacional Autónoma de México, 04510 México, DF, Mexico \\ ${ }^{5}$ PESTIC, Secretaría Académica \& CINVESTAV, IPN, 04430 México DF, Mexico
}

(Received 17 August 1999; revised manuscript received 5 May 2000)

\begin{abstract}
Cooper pairing in two dimensions is analyzed with a set of renormalized equations to determine its binding energy for any fermion number density and all coupling assuming a generic pairwise residual interfermion interaction. Also considered are Cooper pairs (CP's) with nonzero center-of-mass momentum (CMM) and their binding energy is expanded analytically in powers of the CMM up to quadratic terms. A Fermi-sea-dependent linear term in the CMM dominates the pair excitation energy in weak coupling (also called the BCS regime) while the more familiar quadratic term prevails in strong coupling (the Bose regime). The crossover, though strictly unrelated to BCS theory per se, is studied numerically as it is expected to play a central role in a model of superconductivity as a Bose-Einstein condensation of CPs where the transition temperature vanishes for all dimensionality $d \leqslant 2$ for quadratic dispersion, but is nonzero for all $d \geqslant 1$ for linear dispersion.
\end{abstract}

The original Cooper pair (CP) problem $^{1}$ in two (2D) and three (3D) dimensions possesses ultraviolet divergences in momentum space that are usually removed via interactions regularized with large-momentum cutoffs. ${ }^{2}$ One such regularized potential is the BCS model interaction which is of great practical use in studying Cooper pairing ${ }^{1}$ and superconductivity. ${ }^{3}$ Although there are controversies over the precise pairing mechanism, and thus over the microscopic Hamiltonian appropriate for high- $T_{c}$ superconductors, some of the properties of these materials have been explained satisfactorily within a BCS-Bose crossover picture via a $^{4-7}$ ia renormalized BCS theory for a short-range interaction. In the weak-coupling limit of the BCS-Bose crossover description one recovers the pure mean-field BCS theory of weakly bound, severely overlapping CPs. For strong coupling (and/or low density) well separated, nonoverlapping (socalled "local") pairs appear" in what is known as the Bose regime. It is of interest to detail how renormalized Cooper pairing itself evolves independently of the BCS-Bose crossover picture in order to then discuss the possible BoseEinstein (BE) condensation (BEC) of such pairs. We address this here in a single-CP picture, while considering also the important case (generally neglected in BCS theory) of nonzero center-of-mass-momentum (CMM) CPs that are expected to play a significant role in $\mathrm{BE}$ condensates at higher temperatures.

In this report we derive a renormalized Cooper equation for a pair of fermions interacting via either a zero- or a finiterange interaction. We find an analytic expression for the $\mathrm{CP}$ excitation energy up to terms quadratic in the CMM which is valid for any coupling. For weak coupling only the linear term dominates, as it also does for the BCS model interaction. ${ }^{8}$ The linear term was mentioned for 3D as far back as 1964 (Ref. 9, p. 33). For strong coupling we now find that the quadratic term dominates and is just the kinetic energy of the strongly bound composite pair moving in vacuum.
The CP dispersion relation enters into each summand in the $\mathrm{BE}$ distribution function of the boson number equation from which the critical temperature $T_{c}$ of BEC of CPs is extracted. The linear dependence on the CMM of CP binding for weak coupling leads to novel transition properties even in a Heuristic BEC picture of superconductivity ${ }^{10}$ as BEcondensing CPs. It is well known that BEC is possible only for dimensionalities $d>2$ for usual nonrelativistic bosons with quadratic dispersion; this limitation reappears in virtually all BEC schemes thus far applied to describe superconductivity (Refs. 11,12 among others). But for bosons with a linear dispersion relation as found here in weak coupling, BEC can now occur for all $d>1$. Here we discuss the $\mathrm{CP}$ dispersion relation only in $2 \mathrm{D}$. We have also performed a similar analysis in $3 \mathrm{D}$ and obtained the linear to quadratic crossover in the dispersion relation.

Consider a two-fermion system in the CM frame, with each fermion of mass $m$, interacting via the purely attractive short-range separable potential ${ }^{11}$

$$
V_{p q}=-v_{0} g_{p} g_{q}
$$

where $v_{0} \geqslant 0$ is the interaction strength and the $g_{p}$ 's are the dimensionless form factors $g_{p} \equiv\left(1+p^{2} / p_{0}^{2}\right)^{-1 / 2}$, where the parameter $p_{0}$ is the inverse range of the potential so that, e.g., $p_{0} \rightarrow \infty$ implies $g_{p}=1$ and corresponds to the contact or delta potential $V(r)=-v_{0} \delta(\mathbf{r})$. The interaction model (1) mimics a wide variety of possible dynamical mechanisms in superconductors: mediated by phonons, or plasmons, or excitons, or magnons, etc., or even a purely electronic interaction. In the first instance mentioned, two terms of the form (1) can simulate a Coulombic interfermion repulsion surrounded by a longer-ranged electron-phonon attraction. The momentum-space Schrödinger eigenvalue equation for a two-particle bound state in vacuum with binding energy $B_{2}$ $\geqslant 0$ for interaction (1) is ${ }^{5}$ 


$$
\frac{1}{v_{0}}=\sum_{k} \frac{g_{k}^{2}}{B_{2}+\hbar^{2} k^{2} / m}
$$

where $k$ is the wave number in the CM frame and $\hbar^{2} k^{2} / 2 m$ the single-particle energy.

On the other hand, the CP equation for two fermions above the Fermi surface with momenta wave vectors $\mathbf{k}_{1}$ and $\mathbf{k}_{2}$ (and arbitrary CMM wave vector $\mathbf{K} \equiv \mathbf{k}_{1}+\mathbf{k}_{2}$ ) is given by

$$
\left[\frac{\hbar^{2} k^{2}}{m}-E_{K}+\frac{\hbar^{2} K^{2}}{4 m}\right] C_{k}=-\sum_{q}^{\prime} V_{k q} C_{q},
$$

where $\mathbf{k} \equiv \frac{1}{2}\left(\mathbf{k}_{1}-\mathbf{k}_{2}\right)$ is the relative momentum, $E_{K} \equiv 2 E_{F}$ $-\Delta_{K}$ the total pair energy, $\Delta_{K} \geqslant 0$ the CP binding energy, $C_{q} \equiv\langle q \mid \Psi\rangle$ its wave function in momentum space, and the prime on the summation implies restriction to states above the Fermi surface: viz., $|\mathbf{k} \pm \mathbf{K} / 2|>k_{F}$. For the separable interaction (1) Eq. (3) becomes

$$
\sum_{k}^{\prime} \frac{g_{k}^{2}}{\hbar^{2} k^{2} / m+\Delta_{K}-2 E_{F}+\hbar^{2} K^{2} / 4 m}=\frac{1}{v_{0}} .
$$

Although the summand in Eq. (4) is angle-independent, the restriction on the sum arising from the filled Fermi sea is a function of the relative wave vector $\mathbf{k}$, and therefore angle dependent. The potential strength $v_{0}$ can be eliminated between Eqs. (2) and (4) leading to the renormalized CP equation

$$
\sum_{k} \frac{g_{k}^{2}}{B_{2}+\hbar^{2} k^{2} / m}=\sum_{k}^{\prime} \frac{g_{k}^{2}}{\hbar^{2} k^{2} / m+\Delta_{K}-2 E_{F}+\hbar^{2} K^{2} / 4 m} .
$$

Instead of the arbitrary cutoff usually employed in dealing with delta interactions, in Eq. (5) we rely on physical "observables" for the sake of renormalization, viz., the groundstate binding energy $B_{2}$ in vacuum. The sums in Eq. (5) can be transformed to integrals; the restriction in the second term arising from the filled Fermi sea leads to two different expressions depending on whether $\widetilde{K} \equiv K / k_{F}$ is $<2$ or $>2$, as discussed in the Appendix. Letting all variables be dimensionless by expressing them either in units of the Fermi wave number $k_{F}$ or of the Fermi energy $E_{F} \equiv \hbar^{2} k_{F}^{2} / 2 m$, viz., $\xi \equiv k / k_{F}, \widetilde{B}_{2} \equiv B_{2} / E_{F}, \widetilde{\Delta}_{K} \equiv \Delta_{K} / E_{F}$, etc., we define $\alpha_{K}^{2} \equiv 1-\widetilde{\Delta}_{K} / 2-\widetilde{K}^{2} / 4 \equiv-\beta_{K}^{2}$, and $\theta$ the angle between wave vectors $\mathbf{k}$ and $\mathbf{K}$ so that $\xi_{0}(\theta) \equiv \sqrt{1-\widetilde{K}^{2} \sin ^{2} \theta / 4}+\widetilde{K} \cos \theta / 2$ and $\xi_{0}^{\prime}(\theta) \equiv-\sqrt{1-\widetilde{K}^{2} \sin ^{2} \theta / 4}+\widetilde{K} \cos \theta / 2$. For a zero-range interaction, $g_{k}=1$, after some algebra one gets

$$
\begin{gathered}
\int_{0}^{\pi / 2} d \theta \ln \left[\xi_{0}^{2}(\theta)-\alpha_{K}^{2}\right]=\frac{\pi}{2} \ln \frac{\widetilde{B}_{2}}{2}, \quad \widetilde{K}<2, \\
\frac{\pi}{2} \ln \left[\beta_{K}^{2}\right]-\int_{0}^{\theta_{0}} d \theta \ln \frac{\xi_{0}^{\prime 2}(\theta)+\beta_{K}^{2}}{\xi_{0}^{2}(\theta)+\beta_{K}^{2}}=\frac{\pi}{2} \ln \frac{\widetilde{B}_{2}}{2}, \quad \widetilde{K}>2,
\end{gathered}
$$

where $\theta_{0}=\arcsin (2 / \widetilde{K})<\pi / 2$. For $\widetilde{K}=0$ only Eq. (6) applies, in which case $\xi_{0}(\theta)=1, \alpha_{K}^{2} \equiv \alpha_{0}^{2}=1-\widetilde{\Delta}_{0} / 2$ and we obtain the surprising result $\widetilde{\Delta}_{0}=\widetilde{B}_{2}$, i.e., for an attractive delta in- teraction the vacuum and $\mathrm{CP}$ binding energies for zero CMM coincide for all coupling, a result apparently first obtained in Ref. 5. For a nonzero CMM the CP binding energies $\Delta_{K}$ can be calculated from Eqs. (6) and (7). For $\widetilde{K} \neq 0$ a minimum threshold value of $B_{2} / E_{F}$ is found to be required to bind a CP.

Equations (6) and (7) can be solved numerically for the CP binding $\Delta_{K}$ for any CMM. For small CMM only Eq. (6) is relevant; and this equation for small but nonzero $\widetilde{K}$ and for $\widetilde{K}=0$ can be subtracted one from the other. This gives the small-CMM expansion valid for any coupling $B_{2} / E_{F} \geqslant 0$,

$$
\begin{aligned}
\varepsilon_{K} \equiv & \left(\Delta_{0}-\Delta_{K}\right)=\frac{2}{\pi} \hbar v_{F} K \\
& +\left[1-\left\{2-\left(\frac{4}{\pi}\right)^{2}\right\} \frac{E_{F}}{B_{2}}\right] \frac{\hbar^{2} K^{2}}{2(2 m)}+O\left(K^{3}\right),
\end{aligned}
$$

where a nonnegative $C P$ excitation energy $\varepsilon_{K}$ has been defined, and the Fermi velocity $v_{F}$ comes from $E_{F} / k_{F}$ $=\hbar v_{F} / 2$. The leading term in Eq. (8) is linear in the CMM, followed by a quadratic term. The linear $\mathrm{CP}$ dispersion term should not be confused with that of the many-body (collective) excitation spectrum in weak coupling. Only CPs can undergo BEC while bosonic "excitations" (or modes or phonons) cannot since the former are fixed in number while the latter are not. Indeed, the particle-hole [sometimes called the Anderson-Bogoliubov-Higgs $(\mathrm{ABH})]$ modes of excitation energy $\hbar v_{F} K / \sqrt{d}^{13}$ in $d$ dimensions in the zero coupling limit are larger than the weak-coupling $\mathrm{CP}$ dispersion energies $(2 / \pi) \hbar v_{F} K$ and $\frac{1}{2} \hbar v_{F} K$ (Ref. 9, p. 33) in 2D and 3D, ${ }^{9}$ respectively, while in $1 \mathrm{D}$ they happen to coincide-in spite of the fact that $\mathrm{CPs}$ and $\mathrm{ABH}$-like modes are physically distinct entities. The coefficient of the quadratic term in Eq. (8) changes sign at $B_{2} / E_{F}=\Delta_{0} / E_{F} \simeq 0.379$, as one goes from weak $\left(B_{2}=\Delta_{0} \ll E_{F}\right)$ to strong $\left(B_{2}=\Delta_{0} \gg E_{F}\right)$ coupling. If $v_{F}$ (or $\left.E_{F}\right) \rightarrow 0$ explicitly (dilute limit) the first two terms of Eq. (8) reduce simply to

$$
\varepsilon_{K} \rightarrow \frac{\hbar^{2} K^{2}}{2(2 m)}
$$

for any coupling. This is clearly just the familiar nonrelativistic kinetic energy in vacuum of the composite (so-called "local") pair of mass $2 m$ and CMM $K$. The same result (9) is also found to hold in $3 \mathrm{D}$.

Figure 1 shows exact numerical results for the zero-range potential $\left(g_{k}=1\right)$ for different couplings of a CP excitation energy $\varepsilon_{K} / \Delta_{0}$ as function of CMM $K / k_{F}$, both dimensionless. We note that the CPs break up whenever $\Delta_{K}$ turns from positive to negative, i.e., vanishes, or by Eq. (8) when $\varepsilon_{K} / \Delta_{0}=1$. These points are marked in the figure by dots. In addition to the exact results obtained by solving Eqs. (6) and (7), we also exhibit the results for the linear approximation [first term on the right-hand side of Eq. (8), dot-dashed lines, virtually coinciding with the exact curve for all $B_{2} / E_{F}$ $\lesssim 0.1]$ as well as for the quadratic approximation (dashed parabolas) as given by Eq. (9) for stronger couplings. For weak enough coupling or large enough fermion density at any nonzero coupling the exact dispersion relation is virtu- 


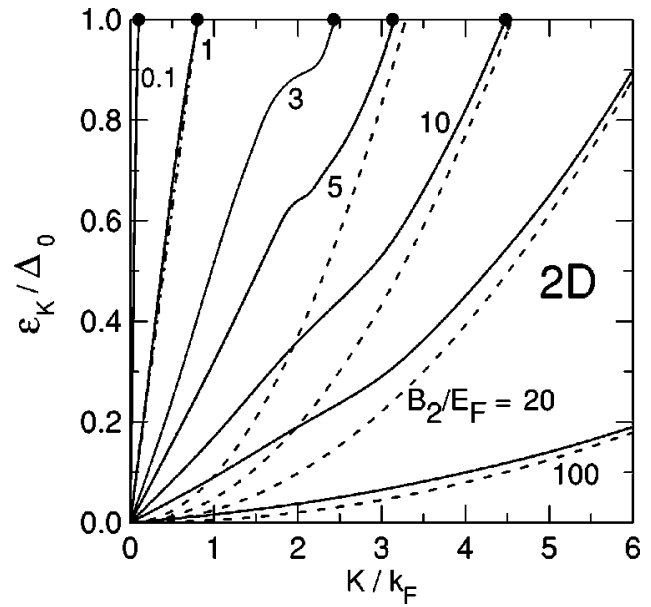

FIG. 1. Dimensionless CP excitation energy $\varepsilon_{K} / \Delta_{0} v s K / k_{F}$, calculated from Eqs. (6) and (7) for different couplings $B_{2} / E_{F}$, full curves. The dot-dashed line is the linear approximation (virtually coincident with the exact curve for $B_{2} / E_{F} \leq 0.1$ ) while the dashed curve is the quadratic term of Eq. (9). Dots denote values of CMM wave number where the CP breaks up, i.e., where $\Delta_{K} \equiv 0$.

ally linear-in spite of the divergence of the isolated quadratic term in Eq. (8) as $B_{2} / E_{F} \rightarrow 0$. As coupling is increased the quadratic dispersion relation (9) slowly begins to dominate. The crossover from a linear to a quadratic dispersion relation manifests itself by a change in curvature from concave down to concave up-these two regions being separated by an inflection point that moves down towards the origin as coupling is increased to infinity.

Figure 2 shows the CP excitation energy $\varepsilon_{K} / \Delta_{0}$ as a function of CMM $K / k_{F}$ calculated for the finite-range interaction form factor $g_{p}=\left(1+p^{2} / p_{0}^{2}\right)^{-1 / 2}$ with $p_{0}=k_{F}$ for weak to moderate coupling. To compare, we also plot the zero-range result as well as the linear relation given by the first term on the right-hand side of Eq. (8). The finite-range curves are closer to the corresponding zero-range ones if labeled by $\Delta_{0} / E_{F}$ instead of by $B_{2} / E_{F}$, as was done with all four sets of curves.

Figure 3 exhibits $\varepsilon_{K} / \Delta_{0}$ as a function of $K / k_{F}$ for the finite-range interaction with $p_{0}=k_{F}$ for stronger couplings. In this case there is no special advantage in labeling the dispersion curves by $\Delta_{0}$ so $B_{2}$ was used with results for $B_{2} / E_{F}=3,10$, and 20 shown. In the zero-range case the curves gradually tend to the quadratic form as $B_{2}$ increases. For finite-range, $p_{0}=k_{F}$, the curves develop a maximum followed by a minimum with a point of inflection in between. The slope at the point of inflection is now negative. Although each curve tends to a quadratic form for large enough $K / k_{F}$, they are quite different from it for small $K / k_{F}$. These "looped" dispersion curves are reminiscent of the "roton" excitation spectrum ${ }^{14}$ in liquid ${ }^{4} \mathrm{He}$.

To summarize, the single $\mathrm{CP}$ problem with nonzero CMM is tracked as it evolves in varying the interfermion shortrange pair interaction from weak to strong or in varying fermion density from high to low, respectively, for any fixed nonzero coupling. The $\mathrm{CP}$ excitation energy is exhibited as a function of its CMM. For weak coupling, the excitation energy is a linear dispersion relation in the CMM, and changes

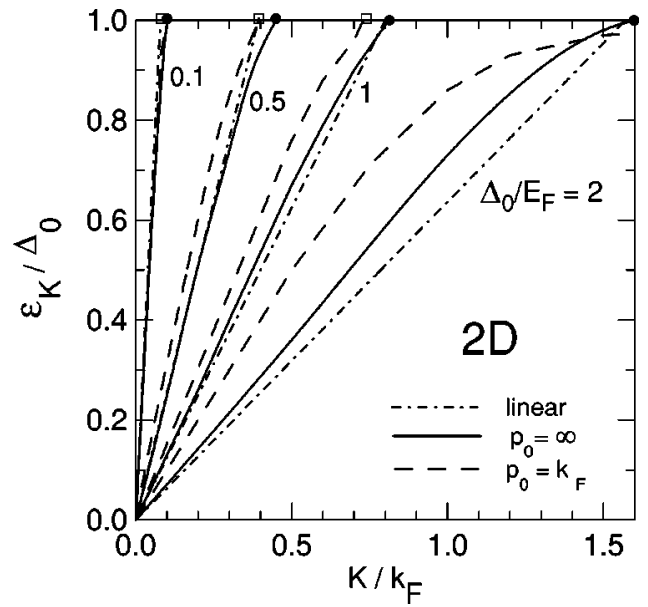

FIG. 2. Same as Fig. 1 but for couplings expressed as $\Delta_{0} / E_{F}$. The dot-dashed line is the linear approximation; the dashed curve is the result for the finite-range interaction $p_{0}=k_{F}$, and the full curve is the zero-range result. For the finite-range potential $\Delta_{0} / E_{F}=0.1$, $0.5,1.0$, and 2.0 correspond to $B_{2} / E_{F}=0.469,1.4,2.45$, and 4 , respectively. Dots and squares mark values of CMM wave number where the $\mathrm{CP}$ breaks up.

very gradually to a quadratic relation as coupling increases. For a zero-range pair interaction in the dispersion curve one typically has a point of inflection with a positive slope separating a region of concave-down curvature for small CMM from a region of concave-up curvature for large CMM. For finite-range pair interactions of sufficiently long range the slope at the point of inflection changes from positive to zero and eventually becomes negative. This leads to maxima and "rotonlike" minima in the CP dispersion curves. These results will play a critical role in a model of superconductivity based on BE condensation of CPs as they will yield, even in $2 \mathrm{D}$ as in the cuprates, BEC transition temperatures $T_{c}$ that interpolate between nonzero values in weak coupling with a

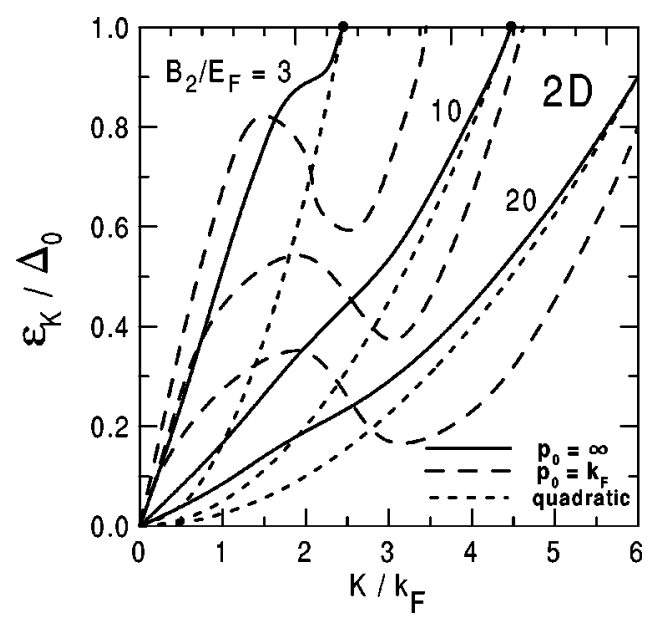

FIG. 3. Same as Fig. 1 but including also finite range at stronger couplings $B_{2} / E_{F}$. The full curve is the exact zero-range result; the short-dashed one the quadratic approximation; the long-dashed one the exact finite-range result with $p_{0}=k_{F}$. Each set of three curves is labeled by different values of $B_{2} / E_{F}$. 
linear CP dispersion relation down to the expected $T_{c} \equiv 0$ value in strong coupling with a quadratic relation.

M.deLl. thanks S. Fujita for discussions, D. M. Eagles for reading the manuscript, and V. V. Tolmachev for extensive correspondence. Partial support from UNAM-DGAPAPAPIIT (México) Grant No. IN102198, CONACyT (México) Grant No. 27828 E, DGES (Spain) Grant No. PB95-0492, and FAPESP (Brazil) is gratefully acknowledged.

\section{APPENDIX}

The restriction that both particles lie above the Fermi sea in Eq. (5) can be written as

$$
\left(\mathbf{k} / k_{F} \pm \mathbf{K} / 2 k_{F}\right)^{2}-1=\xi^{2} \pm \xi \widetilde{K} \cos \theta+\widetilde{K}^{2} / 4-1 \geqslant 0,
$$

where $\xi \equiv k / k_{F}$ and $\widetilde{K} \equiv K / k_{F}$. The equality leads to two pairs of roots in $\xi$, say $\xi_{1,2}=-a \pm b$ and $\xi_{3,4}=a \pm b$, where $a \equiv(\widetilde{K} / 2) \cos \theta, b \equiv \sqrt{1-\left(\widetilde{K}^{2} / 4\right) \sin ^{2} \theta}$, and $\theta$ the angle between $\mathbf{k}$ and $\mathbf{K}$.

For $\widetilde{K}<2, b>a$, one root of the two pairs is positive and the other negative. Thus, Eq. (A1) can be satisfied provided that $\xi>\xi_{1}, \xi_{2}, \xi_{3}, \xi_{4}$, or specifically, if $\xi>\xi_{0}(\theta) \equiv a+b$. For $\widetilde{K}>2$ and $\theta>\theta_{0} \equiv \arcsin (2 / \widetilde{K}), b$ becomes imaginary and Eq. (A1) is satisfied for all $\xi$. Therefore, there is no restriction in the integration over $\xi$. However, for $\widetilde{K}>2$ and $\theta<\theta_{0}, b<a$ the pair of roots $\xi_{1,2}$ are both negative while the pair $\xi_{3,4}$ are both positive (with $\xi_{3}>\xi_{4}$ ). Consequently, in both cases Eq. (A1) is satisfied only if $\xi$ is in the interval $\left[0, \xi_{0}^{\prime}(\theta) \equiv a-b\right]$, and in the interval $\left[\xi_{0}(\theta), \infty\right]$, respectively. Using these restrictions on the $\xi$ integration in Eq. (5) one eventually arrives at Eqs. (6) and (7).
${ }^{1}$ L.N. Cooper, Phys. Rev. 104, 1189 (1956).

${ }^{2}$ S.K. Adhikari and A. Ghosh, J. Phys. A 30, 6553 (1997); S.K. Adhikari and T. Frederico, Phys. Rev. Lett. 74, 4572 (1995).

${ }^{3}$ J. Bardeen, L.N. Cooper, and J.R. Schrieffer, Phys. Rev. 108, 1175 (1957).

${ }^{4}$ D.M. Eagles, Phys. Rev. 186, 456 (1969); A.J. Leggett, J. Phys. Colloq. 41, C7-19 (1980); K. Miyake, Prog. Theor. Phys. 69, 1794 (1983); M. Randeria, J.-M. Duan, and L.-Y. Shieh, Phys. Rev. B 41, 327 (1990).

${ }^{5}$ S.K. Adhikari and A. Ghosh, Phys. Rev. B 55, 1110 (1997).

${ }^{6}$ A. Ghosh and S.K. Adhikari, Eur. Phys. J. B 2, 31 (1998); J. Phys.: Condens. Matter 10, 135 (1998).

${ }^{7}$ M. Casas, J.M. Getino, M. de Llano, A. Puente, R.M. Quick, H. Rubio, and D.M. van der Walt, Phys. Rev. B 50, 15945 (1994); R.M. Carter, M.Casas, J.M. Getino, M. de Llano, A. Puente, H. Rubio, and D.M. van der Walt, ibid. 52, 16149 (1995).
${ }^{8}$ M. Casas, S. Fujita, M. de Llano, A. Puente, A. Rigo, and M.A. Solís, Physica C 295, 93 (1998); S. Fujita and S. Godoy, Quantum Statistical Theory of Superconductivity (Plenum, New York, 1996).

${ }^{9}$ J.R. Schrieffer, Theory of Superconductivity (Benjamin, Reading, MA, 1964).

${ }^{10}$ M. Casas, A. Rigo, M. de Llano, O. Rojo, and M.A. Solís, Phys. Lett. A 245, 55 (1998).

${ }^{11}$ P. Nozières and S. Schmitt-Rink, J. Low Temp. Phys. 59, 195 (1985).

${ }^{12}$ V.V. Tolmachev, Phys. Lett. A 266, 400 (2000); R. Friedberg and T.D. Lee, Phys. Rev. B 40, 6745 (1989); J.M. Blatt, Theory of Superconductivity (Academic, New York, 1964), p. 94, and references therein.

${ }^{13}$ L. Belkhir and M. Randeria, Phys. Rev. B 49, 6829 (1994).

${ }^{14}$ L.D. Landau, J. Phys. (Moscow) 11, 91 (1947); R.P. Feynman, Phys. Rev. 91, 1301 (1953); 94, 262 (1954). 